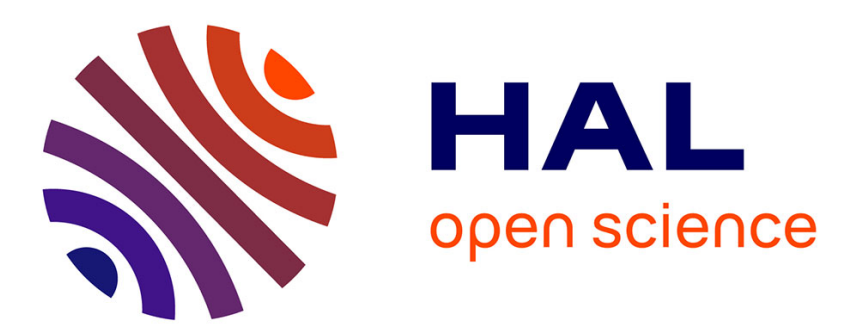

\title{
Existence of Solutions in Continuous-time Optimal Growth Models
}

\author{
Hippolyte d'Albis, Pascal Gourdel, Cuong Le Van
}

\section{To cite this version:}

Hippolyte d'Albis, Pascal Gourdel, Cuong Le Van. Existence of Solutions in Continuous-time Optimal Growth Models. Economic Theory, 2008, 37 (2), pp.321-333. 10.1007/s00199-007-0294-8 . halshs00177269

\section{HAL Id: halshs-00177269 \\ https://shs.hal.science/halshs-00177269}

Submitted on 6 Oct 2007

HAL is a multi-disciplinary open access archive for the deposit and dissemination of scientific research documents, whether they are published or not. The documents may come from teaching and research institutions in France or abroad, or from public or private research centers.
L'archive ouverte pluridisciplinaire HAL, est destinée au dépôt et à la diffusion de documents scientifiques de niveau recherche, publiés ou non, émanant des établissements d'enseignement et de recherche français ou étrangers, des laboratoires publics ou privés. 


\title{
Existence of Solutions in Continuous-time Optimal Growth Models*
}

\author{
Hippolyte d'Albis, Toulouse School of Economics (LERNA), \\ University of Toulouse \\ Pascal Gourdel, CES, University Paris 1, CNRS $^{\dagger}$ \\ Cuong Le Van, CES, University Paris 1, CNRS
}

September 20, 2007

\begin{abstract}
This paper studies the existence of solutions in continuous time optimization problems. It provides a theorem whose conditions can be easily checked in most models of the optimal growth theory, including those with increasing returns and multi-sector economies.
\end{abstract}

Keywords Existence of solutions, optimization, continuous time, optimal growth.

JEL Classification C61, D91, E13.

\section{Introduction}

The modern theory of economic growth hinges on the analysis of optimal allocations of scarce resources over time. To discuss this issue, infinite dimensional optimization techniques in continuous time have been extensively

*The authors thank R. Amir, J.M. Bonnisseau, B. Cornet and S. Demichelis for stimulating comments and suggestions. We are grateful to the referee for her/his valuable comments. The usual disclaimer applies.

†Corresponding author, MSE, 106 Boulevard de l'Hôpital, 75013 Paris, gourdel@univparis1.fr, phone (33)144078296, fax (33)144078301 
used over the last forty years. In most cases the problem may be re-written as the following variational problem:

$$
\begin{array}{l|l}
\max & \int_{0}^{+\infty} G\left(x_{t}, \dot{x}_{t}\right) e^{-r t} d t, \\
\text { s.t. } & \begin{array}{l}
\forall t, \dot{x}_{t} \in \Gamma_{t}\left(x_{t}\right) \\
x_{0} \in \mathbb{R}_{+}^{l} \text { given. }
\end{array}
\end{array}
$$

This problem has a solution provided that $\int_{0}^{+\infty} G\left(x_{t}, \dot{x}_{t}\right) e^{-r t} d t$ exhibits some upper semi-continuity and the correspondence $\Gamma$ some compactness. However, in many papers, those properties are not checked and the existence of the solution is simply assumed. The difficulty is specific to continuous time: to be a feasible path, $x_{t}$ should belong to a ball of $L^{1}$ which is not compact for the $L^{1}$ topology. General results have nevertheless been established. Notably, Magill (1981) provides an existence theorem for problems with a $G$ concave in its arguments. However, his theorem does not apply to the large body of the literature that consider increasing returns. It is true that if the problem is not concave, the difficulty increases since traditional optimality conditions for the Social Planner problem are necessary but not anymore sufficient. Chichilnisky (1981) and Romer (1986a) hence make an important step by providing an existence theorem for optimal solution that apply to models with increasing returns. While proved in a different way, the conditions they proposed require the concavity of $G$ with respect to the one of its arguments which have the highest derivative. Their demonstrations are very nice but the precise set of conditions they propose is rather technical and it is not simple to know if a given economic problem satisfies it. We hence propose an original existence theorem whose conditions are easy to verify in a given economic model. We show this by confronting some well-known models to our existence conditions.

The demonstration we propose extends an initial result of Askenazy and Le Van (1999) about the regularity of the objective function. Then it uses the Dunford-Pettis Criterion to get some sequential compactness for some weak topology and a multi-dimensional version of the Fatou's Lemma to deduce properties for the limit with respect to this topology.

The paper is organized as follows. In section 2, the problem is presented while our theorem is proved in section 3 . In section 4 , we show how our theorem can be easily applied to models of neoclassical growth or endogenous growth. 


\section{The Problem}

Consider the following optimization problem $(\mathcal{P})$ :

$$
\max _{x_{t}, y_{t}} \int_{0}^{+\infty} u\left(x_{t}\right) e^{-r t} d t
$$

subject to:

$$
\begin{aligned}
& \forall t \geq 0, F\left(y_{t}\right) \leq \dot{y}_{t} \leq G\left(\bar{y}_{t}, y_{t}, x_{t}\right), \\
& x_{t} \in \mathbb{R}_{+}^{C}, y_{t} \in \mathbb{R}_{+}^{K}, \\
& y_{0} \in \mathbb{R}_{+}^{K}, r>0 \text { are given. }
\end{aligned}
$$

In this setting, $x_{t}$ is the control variable, $y_{t}$ is the state variable, $\dot{y}_{t}$ its derivative with respect to time (the definition of $\dot{y}_{t}$ will be more precise in Remark 1). and $\bar{y}_{t} \in \mathbb{R}_{+}^{K}$ is a given externality. The sets $C$ and $K$ are finite; for simplicity, $\left(y^{1}, \ldots, y^{K}\right)$ denote elements of $\mathbb{R}^{K}$ and $\left(x^{1}, \ldots, x^{C}\right)$ elements of $\mathbb{R}^{C}$.

Assume:

A1. The functions $F$ (respectively $G$ ) are continuous on $\mathbb{R}_{+}^{K}$ (respectively on $\mathbb{R}_{+}^{K} \times \mathbb{R}_{+}^{K} \times \mathbb{R}_{+}^{C}$ ).

A2. For any $j \in K$, the function $G^{j}$ is concave with respect to $x_{t}$.

A3. There exist $\left(b_{i} \geq 0, A_{i}>0, i=1, \ldots, C\right)$ and $\left(a_{j} \geq 0, A_{j}^{\prime}>0\right.$, $j=1, \ldots, K)$ such that: $\forall t, \forall j \in K, \bar{y}_{t}^{j} \leq A_{j}^{\prime} e^{a_{j} t}$ and if $x$, and $y$ satisfy the following differential constraint

$\forall t, F\left(y_{t}\right) \leq \dot{y}_{t} \leq G\left(\bar{y}_{t}, y_{t}, x_{t}\right)$, then $\forall t,\left\{\begin{array}{l}\forall j \in K, y_{t}^{j} \leq A_{j}^{\prime} e^{a_{j} t}, \text { and }\left|\dot{y}_{t}^{j}\right| \leq A_{j}^{\prime} e^{a_{j} t}, \\ \forall i \in C, x_{t}^{i} \leq A_{i} e^{b_{i} t} .\end{array}\right.$

A4. $r>\sup \left\{b_{i}, i \in C\right\}$.

A5. The function $u$ is concave, non decreasing and upper semi-continuous ${ }^{1}$ from $\mathbb{R}_{+}^{C}$ into $\mathbb{R} \cup\{-\infty\}$ and finite valued on $\mathbb{R}_{++}^{C}$.

Remark 1 Observe that under $\boldsymbol{A} 3$ and $\boldsymbol{A} 4$, the functions $y^{j} e^{-\rho t}$ belong to the Sobolev space $W^{1,1}\left(\mathbb{R}_{+}\right)$, while the functions $\bar{y}^{j}$ are in $L^{1}\left(e^{-\rho t} d t\right)$, with some $\rho>\max \left\{a_{j}: j \in K\right\}$ and $x^{i}$ are in $L^{1}\left(e^{-r t} d t\right)$.

\footnotetext{
${ }^{1}$ The extended real line $\overline{\mathbb{R}}=[-\infty,+\infty]$ will always be endowed with its usual topology cf. for example Rudin (1987).
} 
Assumption A5 allows for utility functions unbounded from below, such as $u(c)=\log (c)$ or $u(c)=c^{\sigma} / \sigma$ with $\sigma<0$ that are extensively used in economic models. Note that this does not imply the continuity of $u$. Indeed, let us consider in the case where $C=2$, and the function

$$
u\left(c_{1}, c_{2}\right)=\left\{\begin{array}{cll}
-\frac{\left(c_{2}-1\right)^{2}}{2 c_{1}} & \text { if } c_{1}>0 \text { and } c_{2}<1 \\
-\infty & \text { if } c_{1}=0 \text { and } c_{2}<1 \\
0 & \text { if } c_{2} \geq 1
\end{array}\right.
$$

It is easy to show that $u$ satisfies $\mathbf{A} 5$ but is not continuous at point $(0,1)$. The hessian matrix is equal to

$$
\left(\begin{array}{cc}
-\frac{\left(c_{2}-1\right)^{2}}{\left(c_{1}\right)^{3}} & \frac{\left(c_{2}-1\right)}{\left(c_{1}\right)^{2}} \\
\frac{\left(c_{2}-1\right)}{\left(c_{1}\right)^{2}} & \frac{-1}{c_{1}}
\end{array}\right)
$$

on $] 0,+\infty[\times[0,1[$. The determinant is equal to zero and the other eigenvalue is negative.

We can state the following lemma which extends the corresponding result of Askenazy and Le Van (1999).

Lemma 1 Under A5, the function $L_{+}^{1}\left(e^{-r t} d t\right) \rightarrow \mathbb{R} \cup\{-\infty\}$ defined by $x \rightarrow$ $\int_{0}^{+\infty} u\left(x_{t}\right) e^{-r t} d t$ is upper semi-continuous for the topology $\sigma\left(L^{1}\left(e^{-r t} d t\right), L^{\infty}\right)$, and the usual topology on $\overline{\mathbb{R}}$.

Proof. We will prove that the function $x \in L_{+}^{1}\left(e^{-r t} d t\right) \rightarrow \int_{0}^{+\infty} u\left(x_{t}\right) e^{-r t} d t$ is upper semi-continuous for the $L^{1}$-topology. Since it is concave, it will therefore be upper semi-continuous for the topology $\sigma\left(L^{1}\left(e^{-r t} d t\right), L^{\infty}\right)$.

The proof will be done in several steps.

Step 1

Claim The function takes values in $\mathbb{R} \cup\{-\infty\}$.

Proof Indeed, let $x \in L^{1}\left(e^{-r t} d t\right)$, and let us define $T_{0}=\{t: u(x(t)) \geq 0\}$. Since, for $a>0$, we can choose $p(a) \in \partial u(a)$ (the non-empty superdifferential), this allows to write for all $t \geq 0, u(a)-u(x(t)) \geq p(a)(a-x(t))$. Hence,

$$
\int_{T_{0}} u(x(t)) e^{-r t} d t \leq(u(a)-a p(a)) \int_{T_{0}} e^{-r t} d t+p(a) \int_{T} x(t) e^{-r t} d t<+\infty
$$


This implies in particular, $\int_{0}^{+\infty} u(x(t)) e^{-r t} d t$ exists in $\overline{\mathbb{R}}$ and is different of $+\infty$.

Step 2

Claim Let $x \in L^{1}\left(e^{-r t} d t\right)$. Then

$$
\int_{0}^{+\infty} u(x(t)) e^{-r t} d t \in \mathbb{R} \Leftrightarrow u(x(t)) \in L^{1}\left(e^{-r t}\right) .
$$

Proof Let us define the measurable sets, $T_{0}=\{t: u(x(t)) \geq 0\}$ and $T_{0}^{\prime}=$ $\{t: u(x(t))<0\}$. It suffice to recall that the finiteness of $\int_{T_{0}} u(x(t)) e^{-r t} d t$ allows us to write (in $\overline{\mathbb{R}}$ )

$$
\left\{\begin{array}{l}
\int_{0}^{+\infty}|u(x(t))| e^{-r t} d t=\int_{T_{0}} u(x(t)) e^{-r t} d t-\int_{T_{0}^{\prime}} u(x(t)) e^{-r t} d t \\
\int_{0}^{+\infty} u(x(t)) e^{-r t} d t=\int_{T_{0}} u(x(t)) e^{-r t} d t+\int_{T_{0}^{\prime}} u(x(t)) e^{-r t} d t .
\end{array}\right.
$$

This proves the second claim.

Step 3: Proof of the lemma.

Let $\left(x^{n}\right)$ be a nonnegative sequence of functions which converge to $x$ in $L^{1}$. We want to prove in $\overline{\mathbb{R}}$ that $\lim \sup _{n} \int_{0}^{+\infty} u\left(x^{n}(t)\right) e^{-r t} d t \leq \int_{0}^{+\infty} u(x(t)) e^{-r t} d t$. Let us denote $M=\limsup _{n} \int_{0}^{+\infty} u\left(x^{n}(t)\right) e^{-r t} d t$. If $M=-\infty$, the proof is over.

So assume $M \in \mathbb{R}$. Let us consider $\left(x^{n_{k}}\right)$ be a subsequence of $\left(x^{n}\right)$ such that $M=\lim _{k} \int_{0}^{+\infty} u\left(x^{n_{k}}(t)\right) e^{-r t} d t$. Note that from Step 2, $u\left(x^{n_{k}}\right) \in$ $L^{1}\left(e^{-r t} d t\right)$ for any $k$ large enough. From Theorem IV.9 in Brezis (1987) (or Theorem 3.12 in Rudin (1987)), one can assume that $x^{n}$ converges to $x$ pointwise a.e. Let us fix $a \in \mathbb{R}_{++}$and $p \in \partial u(a)$. We can introduce the non-positive functions

$$
\forall t, f_{n}(t)=u\left(x^{n}(t)\right)-p a+u(a)-p x^{n}(t) \leq 0 .
$$

From Fatou's Lemma (see Rudin (1987)), we get

$$
\begin{aligned}
\underset{k}{\limsup \int_{0}^{+\infty} f_{n_{k}}(t) e^{-r t} d t} \leq \int_{0}^{+\infty} \limsup _{k} f_{n_{k}}(t) e^{-r t} d t \\
\leq \int_{0}^{+\infty} \limsup _{k}\left(u\left(x_{n_{k}}(t)\right)-p a+u(a)-p x_{n_{k}}(t)\right) e^{-r t} d t
\end{aligned}
$$

In view of the pointwise convergence and the upper semi-continuity of $u$, we deduce

$$
\underset{k}{\limsup } \int_{0}^{+\infty} f_{n_{k}}(t) e^{-r t} d t \leq \int_{0}^{+\infty}(u(x(t))-p a+u(a)-p x(t)) e^{-r t} d t
$$


This can be equivalently rewritten as

$$
\underset{k}{\limsup } \int_{0}^{+\infty}\left(u\left(x^{n_{k}}(t)\right)-p x^{n_{k}}(t)\right) e^{-r t} d t \leq \int_{0}^{+\infty}(u(x(t))-p x(t)) e^{-r t} d t
$$

Since $\lim _{k} \int_{0}^{+\infty} x^{n_{k}}(t) e^{-r t} d t=\int_{0}^{+\infty} x(t) e^{-r t} d t$, we deduce finally that $M \leq$ $\int_{0}^{+\infty} u(x(t)) e^{-r t} d t$ and the proof is complete.

Remark 2 If $u(0)=0$, then the function $x \in L_{+}^{1}\left(e^{-r t} d t\right) \rightarrow \int_{0}^{+\infty} u(x(t)) e^{-r t} d t$ is actually continuous for the $L^{1}$-topology. Indeed, first notice that since $u$ is non decreasing, $u(0)$ finite implies that $u$ is bounded away from below by $u(0)$. We can now apply Theorem 10.2 of Rockafellar (1970) in order to deduce that $u$ is lower semi-continuous, hence continuous on $\mathbb{R}_{+}^{C}$. Since $x^{n} \rightarrow x$ in $L^{1}\left(e^{-r t} d t\right)$ when $n$ goes to infinity, from Theorem IV.9 in Brezis (1987) (or Theorem 3.12 in Rudin (1987)), one can assume that $x^{n}$ converges to $x$ pointwise a.e. Consequently, $u\left(x^{n}\right)$ converges to $u(x)$ pointwise a.e. Applying again Fatou's Lemma, we get

$\int_{0}^{+\infty} u(x(t)) e^{-r t} d t=\int_{0}^{+\infty} \liminf _{n \rightarrow+\infty} u\left(x^{n}(t)\right) e^{-r t} d t \leq \liminf _{n \rightarrow+\infty} \int_{0}^{+\infty} u\left(x^{n}(t)\right) e^{-r t} d t$.

We have proved that the function $x \in L_{+}^{1}\left(e^{-r t} d t\right) \rightarrow \int_{0}^{+\infty} u(x(t)) e^{-r t} d t$ is lower semi-continuous. From Lemma 1, it is therefore continuous.

\section{Existence of Solutions}

This section provides our main result:

Theorem 1 Under assumptions $A 1, \ldots, A 5$, if the value of Problem $(\mathcal{P})$ is finite, then $(\mathcal{P})$ has a solution.

Proof. First note that the variables $\left(x_{t}, y_{t}\right)$ will be said feasible if they satisfy the constraints of Problem $(\mathcal{P})$. Observe that assumptions $A 3$ and A4 imply that $\int_{0}^{+\infty} u\left(x_{t}\right) e^{-r t} d t$ is uniformly bounded from above, on the set of feasible controls $x_{t}$. Let the sequence $\left(x^{n}\right)$ satisfy $\lim _{n \rightarrow \infty} \int_{0}^{+\infty} u\left(x_{t}^{n}\right) e^{-r t} d t$ $=M \stackrel{\text { def }}{=} \sup \int_{0}^{+\infty} u\left(x_{t}\right) e^{-r t} d t$ over the set of feasible controls. Assumptions $A 3$ and $A 4$ guaranty that $x^{n}$ are elements of $L_{+}^{1}\left(e^{-r t} d t\right)$. Assumption $A 3$ 
implies that the sequence satisfies the Dunford-Pettis criterion ${ }^{2}$ and has a subsequence, denoted also $\left(x^{n}\right)$ for simplicity, which converges to $x^{*} \in L_{+}^{1}$ for the topology $\sigma\left(L^{1}\left(e^{-r t} d t\right), L^{\infty}\right)$. From Lemma $1, \int_{0}^{+\infty} u\left(x_{t}^{*}\right) e^{-r t} d t \geq M$. To end the proof, it remains to show that $x^{*}$ is feasible.

Observe first that for a defined $\rho>\max \left\{a_{j}: j \in K\right\}$ then the associated sequences $\left(y^{n}, \dot{y}^{n}\right)$ also satisfy the Dunford-Pettis criterion for $L^{1}\left(e^{-\rho t} d t\right)$. It can therefore be assumed that they converge respectively to $y^{*}, z^{*}$ for the topology $\sigma\left(L^{1}\left(e^{-\rho t} d t\right), L^{\infty}\right)$. Then for all $t$ :

$$
\int_{0}^{t} \dot{y}^{n}(s) d s \rightarrow \varphi(t) \stackrel{\text { def }}{=} \int_{0}^{t} z^{*}(s) d s .
$$

In particular, $y^{n}(t) \rightarrow \varphi(t)+y_{0}$, for every almost $t$. Since $\forall t, \forall j, 0 \leq$ $y^{n, j}(t) \leq A_{j}^{\prime} e^{a_{j} t} \in L^{1}\left(e^{-r t} d t\right)$, use Lebesgue Theorem to obtain that $y^{n}$ converges to $\varphi+y_{0}$ for the strong topology (and consequently for the weak topology) of $L^{1}\left(e^{-\rho t} d t\right)$. Thus $y^{*}=\varphi+y_{0}$ and $\dot{y}^{*}=z^{*}$.

Using the multidimensional Fatou's Lemma (see Appendix), given $t$, there exist $\left(\theta_{t k}\right)_{k=1, \ldots, K+C+1}$ such that $\sum_{k=1}^{K+C+1} \theta_{t k}=1, \theta_{t k} \geq 0, \forall k$, and

$$
\left(\dot{y}_{t}^{*}, x_{t}^{*}\right)=\sum_{k=1}^{K+C+1} \theta_{t k}\left(\varsigma_{t k}^{1}, \varsigma_{t k}^{2}\right)
$$

where $\left(\varsigma_{t k}^{1}, \varsigma_{t k}^{2}\right) \in a c\left(\dot{y}_{t}^{n}, x_{t}^{n}\right)$ the set of cluster points of the sequence $\left(\dot{y}_{t}^{n}, x_{t}^{n}\right)$. Given $(t, k)$, there will be some increasing function $\phi_{t k}: \mathbb{N} \rightarrow \mathbb{N}$ such that

$$
\left(\dot{y}_{t}^{\phi_{t k}(n)}, x_{t}^{\phi_{t k}(n)}\right) \underset{n \rightarrow+\infty}{\longrightarrow}\left(\varsigma_{t k}^{1}, \varsigma_{t k}^{2}\right) .
$$

Then, for all $j \in K$

$\dot{y}_{t}^{* j}=\sum_{k=1}^{K+C+1} \theta_{t k} \varsigma_{t k}^{1 j}=\sum_{k=1}^{K+C+1} \theta_{t k} \lim _{n \rightarrow+\infty} \dot{y}_{t}^{j, \phi_{t k}(n)} \leq \sum_{k=1}^{K+C+1} \theta_{t k} \lim _{n \rightarrow+\infty} G^{j}\left(\bar{y}_{t}, y_{t}^{\phi_{t k}(n)}, x_{t}^{\phi_{t k}(n)}\right)$.

Since $G$ is continuous and $y^{n}$ converges pointwise, it yields:

$$
\dot{y}_{t}^{* j} \leq \sum_{k=1}^{K+C+1} \theta_{t k} G^{j}\left(\bar{y}_{t}, \lim _{n \rightarrow+\infty} y_{t}^{\phi_{t k}(n)}, \lim _{n \rightarrow+\infty} x_{t}^{\phi_{t k}(n)}\right)=\sum_{k=1}^{K+C+1} \theta_{t k} G^{j}\left(\bar{y}_{t}, y_{t}^{*}, \varsigma_{t k}^{2}\right) .
$$

\footnotetext{
${ }^{2}$ See Dunford and Schwartz, 1967, corollary 11, p. 294
} 
From A2,

$$
\dot{y}_{t}^{* j} \leq G^{j}\left(\bar{y}_{t}, y_{t}^{*}, \sum_{k=1}^{K+C+1} \theta_{t k} \varsigma_{t k}^{2}\right)=G^{j}\left(\bar{y}_{t}, y_{t}^{*}, x_{t}^{*}\right)
$$

Recall that

$$
\dot{y}_{t}^{* j}=\sum_{k=1}^{K+C+1} \theta_{t k} \varsigma_{t k}^{1 j}=\sum_{k=1}^{K+C+1} \theta_{t k} \lim _{n \rightarrow+\infty} y_{t}^{j, \phi_{t k}(n)} \geq \sum_{k=1}^{K+C+1} \theta_{t k} \lim _{n \rightarrow+\infty} F^{j}\left(y_{t}^{\phi_{t k}(n)}\right) \text {. }
$$

Since $F$ is continuous and $y^{n}$ converges pointwise, it yields:

$$
\dot{y}_{t}^{* j} \geq \sum_{k=1}^{K+C+1} \theta_{t k} F^{j}\left(\lim _{n \rightarrow+\infty} y_{t}^{\phi_{t k}(n)}\right)=\sum_{k=1}^{K+C+1} \theta_{t k} F^{j}\left(y_{t}\right)=F^{j}\left(y_{t}\right) .
$$

Thus, $\left(y^{*}, x^{*}\right)$ have been proved to be feasible. Hence $M=\sup \int_{0}^{+\infty} u\left(x_{t}\right) e^{-r t} d t=$ $\int_{0}^{+\infty} u\left(x_{t}^{*}\right) e^{-r t} d t$.

One can use the proof of the previous theorem to get the following corollary.

Corollary 1 Assume A1, A2, A3, A5. Then the following finite horizon problem has a solution.

$$
\max _{x_{t}, y_{t}} \int_{0}^{T} u\left(x_{t}\right) e^{-r t} d t
$$

subject to:

$$
\begin{aligned}
& \forall t \in[0, T], F\left(y_{t}\right) \leq \dot{y}_{t} \leq G\left(\bar{y}_{t}, y_{t}, x_{t}\right), \\
& x_{t} \in \mathbb{R}_{+}^{C}, y_{t} \in \mathbb{R}_{+}^{K}, \\
& \left.y_{0} \in \mathbb{R}_{+}^{K}, r>0, T \in\right] 0,+\infty[\text { are given } .
\end{aligned}
$$

Remark 3 Let us consider the Social Planner Problem associated with $(\mathcal{P})$.

$$
\max _{x_{t}, y_{t}} \int_{0}^{+\infty} u\left(x_{t}\right) e^{-r t} d t
$$

subject to:

$$
\begin{aligned}
& \forall t \geq 0, F\left(y_{t}\right) \leq \dot{y}_{t} \leq G\left(y_{t}, y_{t}, x_{t}\right)=\widetilde{G}(\bar{y}, y, x), \\
& x_{t} \in \mathbb{R}_{+}^{C}, y_{t} \in \mathbb{R}_{+}^{K}, \\
& y_{0} \in \mathbb{R}_{+}^{K}, r>0 \text { are given, }
\end{aligned}
$$

where $\widetilde{G}(\bar{y}, y, x)=\Phi(\bar{y}) G(y, y, x), \Phi$ is the constant function equal to 1 . The Social Planner Problem is of the same type than $(\mathcal{P})$ where $G$ is replaced by $\widetilde{G}$. 


\section{Applications}

In all the following examples, the finiteness of the value is obvious.

\subsection{The Ramsey model with discounting}

Consider the model developed by Cass (1965) and Koopmans (1965):

$$
\max _{c_{t}, k_{t}} \int_{0}^{+\infty} u\left(c_{t}\right) e^{-r t} d t
$$

subject to $\forall t \geq 0$,

$$
\begin{aligned}
& -\delta k_{t} \leq \dot{k}_{t} \leq f\left(k_{t}\right)-\delta k_{t}-c_{t} \\
& c_{t} \geq 0, k_{t} \geq 0 \\
& \delta \geq 0, k_{0}>0, r>0 \text { are given. }
\end{aligned}
$$

Variables $c_{t}$ and $k_{t}$ denote respectively the consumption and the capital stock at time $t$. It is supposed that the instantaneous utility function $u$ satisfies assumption $A 5$, and that the production function $f$ is concave, continuous, increasing, differentiable, and satisfies $f(0)=0, f^{\prime}(\infty)=0$.

Define $x_{t}=c_{t}, y_{t}=k_{t}, \bar{y}_{t}=1, G\left(\bar{y}_{t}, y_{t}, x_{t}\right)=\bar{y}_{t}\left(f\left(y_{t}\right)-\delta y_{t}-x_{t}\right), F\left(y_{t}\right)=$ $-\delta y_{t}$. Now, check whether Assumptions $A 1, \ldots, A 4$ are satisfied.

$A 1$ : It is obvious that $F$ and $G$ are continuous.

A2: It is also obviously satisfied.

A3: Since $f^{\prime}(\infty)=0$, for any $\varepsilon \in(0, r)$, there exists $B$ such that $\forall y \geq 0$, $f(y) \leq B+\varepsilon y$. It is then easy to show that there exists $A^{\prime \prime}>0$, such that $\forall t \geq 0, y_{t} \leq A^{\prime \prime} e^{\varepsilon t}$. Using $-\delta y_{t} \leq \dot{y}_{t}$, this implies $\forall t \geq 0, x_{t} \leq A e^{\varepsilon t}$ with $A=B+\varepsilon A^{\prime \prime}$, and $\left|\dot{y}_{t}\right| \leq A^{\prime} e^{\varepsilon t}$ with $A^{\prime}=\max \left\{\delta k_{0}, B+\varepsilon A^{\prime \prime}\right\}$.

A4: is automatically satisfied.

Observe that the function $k e^{-r t}$ is in $W^{1,1}\left(\mathbb{R}_{+}\right)$, and $c$ is in $L^{1}\left(e^{-r t} d t\right)$.

\subsection{The Ramsey model with endogenous labor}

Consider now the following problem:

$$
\max _{c_{t}, l_{t}, k_{t}} \int_{0}^{+\infty} u\left(c_{t}, l_{t}\right) e^{-r t} d t
$$


subject to $\forall t \geq 0$

$$
\begin{aligned}
& -\delta k_{t} \leq \dot{k}_{t} \leq f\left(k_{t}, 1-l_{t}\right)-\delta k_{t}-c_{t}, \\
& c_{t} \geq 0, k_{t} \geq 0,0 \leq l_{t} \leq 1, \\
& \delta \geq 0, k_{0}>0, r>0 \text { are given. }
\end{aligned}
$$

The variable $l_{t}$ denotes the leisure at time $t$. It is supposed that the utility function $u$ satisfies $A 5$ and that the production function $f$ is concave, increasing in both arguments, continuously differentiable, and satisfies $f(0, l)=$ $f(k, 0)=0$, and $f^{\prime}(\infty, l)=0$.

As in the previous example, one can find some $\varepsilon$ in $] 0, r[$ such that for any $t \geq 0, c_{t} \leq A e^{\varepsilon t}, k_{t} \leq A^{\prime} e^{\varepsilon t},\left|\dot{k}_{t}\right| \leq A^{\prime} e^{\varepsilon t}$. Denote $x_{t}=\left(c_{t}, l_{t}\right), y_{t}=k_{t}$, $\bar{y}_{t}=1, G\left(\bar{y}_{t}, y_{t}, x_{t}\right)=\bar{y}_{t}\left(f\left(y_{t}\right)-\delta y_{t}-x_{t}\right), F\left(y_{t}\right)=-\delta y_{t}$. It is then easy to check that Assumptions $A 1, \ldots, A_{4}$ are satisfied.

In this model, again the function $k e^{-r t}$ is in $W^{1,1}\left(\mathbb{R}_{+}\right)$, and $c$ is in $L^{1}\left(e^{-r t} d t\right)$. Obviously, $l$ is in $L^{\infty}$.

\subsection{Endogenous growth}

Consider the Romer (1986b) Model. For a given path $\bar{k}_{t} \geq 0$, solve:

$$
\max _{c_{t}, k_{t}} \int_{0}^{+\infty} u\left(c_{t}\right) e^{-r t} d t
$$

subject to $\forall t \geq 0$

$$
\begin{aligned}
& c_{t}+i_{t} \leq F\left(\bar{k}_{t}, k_{t}\right), \\
& \dot{k}_{t}=g\left(\frac{i_{t}}{k_{t}}\right), \\
& c_{t} \geq 0, \quad i_{t} \geq 0, k_{t} \geq 0, \\
& k_{0}>0, \quad r>0 \text { are given. }
\end{aligned}
$$

Variables $i_{t}, k_{t}, c_{t}$ respectively denote the investment, the stock of knowledge and the consumption at time $t$. It is supposed that the function $g$ is concave, increasing, and satisfies $g(0)=0, g(\infty)=\lambda>0$. Assume, moreover, that $F$ is concave, nonnegative, and satisfies $F(y, k) \leq y^{\alpha} k^{\beta}$ for every $(y, k) \geq 0$, and $\alpha>0, \beta>0$. The function $u$, as previously, satisfies Assumption $A 5$.

Define:

$$
\begin{gathered}
G(\bar{y}, y, x)=y g\left(\frac{F(\bar{y}, y)-x}{y}\right) \text { when } y>0 \\
F(\bar{y}, y)-x \geq 0
\end{gathered}
$$


and:

$$
G(\bar{y}, 0,0)=0 .
$$

When $x_{t}=c_{t}, y_{t}=k_{t}$, the problem is then equivalent to:

$$
\max _{c_{t}, k_{t}} \int_{0}^{+\infty} u\left(c_{t}\right) e^{-r t} d t
$$

subject to $\forall t \geq 0$,

$$
\begin{aligned}
& 0 \leq \dot{k}_{t} \leq G\left(\bar{k}_{t}, k_{t}, c_{t}\right), k_{t} \geq 0, \\
& k_{0}>0, r>0 \text { are given. }
\end{aligned}
$$

We assume furthermore that

(i) $\sup _{t \in \mathbb{R}_{+}}\left[\frac{\bar{y}_{t}}{e^{\lambda t}}\right]<+\infty$,

(ii) $r>\lambda(\alpha+\beta)$.

This condition ensures that our objective function is finite valued. This also implies that the maximal growth rate of the output (equal to $\lambda(\alpha+\beta)$ ) must be less than the discount rate $r$.

Now, check whether Assumptions $A 1, \ldots, A 4$ are fulfilled.

$A 1$ : It is easy to see that $G$ is continuous.

A2: It is obvious that $G$ is concave in $x$.

A3: Since $0 \leq \dot{y}_{t} \leq \lambda y_{t}$, it implies that $\forall t, y_{t} \leq k_{0} e^{\lambda t},\left|\dot{y}_{t}\right| \leq \lambda k_{0} e^{\lambda t}$ and $x_{t} \leq \bar{k}_{0} k_{0} e^{\lambda(\alpha+\beta) t}$.

A4: It is satisfied from the assumption $r>\lambda(\alpha+\beta)$ stated above.

In this model, the functions $k e^{-\rho t}$ belong to the Sobolev space $W^{1,1}\left(\mathbb{R}_{+}\right)$, with $\rho>\lambda, c$ is in $L^{1}\left(e^{-r t} d t\right)$.

\subsection{Endogenous growth with human capital}

Consider finally the Lucas (1988) Model. For a given human capital path $\left(\bar{h}_{t}\right)$, solve:

$$
\max _{c_{t}, \theta_{t}, k_{t}, h_{t}} \int_{0}^{+\infty} u\left(c_{t}\right) e^{-r t} d t
$$

subject to: $\forall t \geq 0$,

$$
\begin{aligned}
& -\delta k_{t} \leq \dot{k}_{t} \leq k_{t}^{\alpha}\left(\theta_{t} h_{t}\right)^{1-\alpha} \bar{h}_{t}^{\gamma}-\delta k_{t}-c_{t}, \\
& 0 \leq \dot{h}_{t} \leq \phi\left(1-\theta_{t}\right) h_{t} \\
& c_{t} \geq 0, k_{t} \geq 0,0 \leq h_{t}, 0 \leq \theta_{t} \leq 1, \\
& k_{0}>0, h_{0}>0 \text { are given, } \\
& r>0,0<\alpha<1, \gamma>0 .
\end{aligned}
$$


Variables $c_{t}, \theta_{t}, k_{t}$ and $h_{t}$ denote respectively the consumption, the working time, the physical capital and the human capital at time $t$. Moreover, it is supposed that function $\phi$ is increasing, concave and satisfies $\phi(0)=0$, $\phi(1)=\mu>0$ while function $u$ is concave and increasing.

Define $x_{t}=\left(c_{t}, \theta_{t}\right), y_{t}=\left(k_{t}, h_{t}\right), \bar{y}_{t}=\left(1, \bar{h}_{t}\right), u(x)=v(c), F(y)=(-\delta k, 0)$, $G\left(\bar{y}_{t}, y_{t}, x_{t}\right)=k_{t}^{\alpha}\left(\theta_{t} h_{t}\right)^{1-\alpha} \bar{h}_{t}^{\gamma}-\delta k_{t}-c_{t}$.

Assume furthermore that

(i) $\sup _{t \in \mathbb{R}_{+}}\left[\frac{\bar{y}_{t}}{e^{\mu t}}\right]<+\infty$,

(ii) $r>\frac{\mu(1-\alpha+\gamma)}{(1-\alpha)}$.

Obviously, Assumption $A 5$ is satisfied. Now, check A1, ..,A4.

A1: It is obviously true.

A2: It is immediate since $0<\alpha<1$

A3: It follows from the dynamic constraint on human capital that $\left|\dot{h}_{t}\right| \leq$ $\mu h_{0} e^{\mu t}, h_{t} \leq h_{0} e^{\mu t}$. From the constraints on physical capital, one obtains that $k_{t} \leq\left(\frac{h_{0}(1-\alpha)}{\mu(1-\alpha+\gamma)}\right)^{\frac{1}{1-\alpha}} e^{\frac{\mu(1-\alpha+\gamma) t}{(1-\alpha)}}, c_{t} \leq\left(\frac{h_{0}(1-\alpha)}{\mu(1-\alpha+\gamma)}\right)^{\frac{1}{1-\alpha}} h_{0}^{1-\alpha+\mu} e^{\frac{\mu(1-\alpha+\gamma) t}{(1-\alpha)}},\left|\dot{k}_{t}\right| \leq$ $\max \left\{h_{0}^{\frac{1}{1-\alpha}}\left(\frac{(1-\alpha)}{\mu(1-\alpha+\gamma)}\right)^{\frac{\alpha}{1-\alpha}}, \delta k_{0}\right\} e^{\frac{\mu(1-\alpha+\gamma) t}{(1-\alpha)}}$.

Condition (ii) ensures that our objective function is finite valued. It also implies that the maximal growth rate of the output (equal to $\left.\frac{\mu(1-\alpha+\gamma)}{(1-\alpha)}\right)$ must be less than the discount rate $r$. Thanks to this additional assumption, $A 4$ is satisfied.

In this model, the function $h e^{-\rho t}$ belongs to the Sobolev space $W^{1,1}\left(\mathbb{R}_{+}\right)$, with $\rho>\mu$ while $c$ and $k$ are in $L^{1}\left(e^{-r t} d t\right)$.

\section{Conclusion}

This paper has proposed an original theorem to prove the existence of an optimal solution for most macroeconomic problems. The next step would be to prove the existence of an equilibrium with externality $\bar{y}$ defined endogenously. This equilibrium can be viewed as a fixed-point but proving the existence is however a difficult task. Let us introduce the correspondence of "compatibility" which plays a role analogous to the budget correspondence.

$$
\Delta(\bar{y})=\left\{(x, y) \mid y(0)=y_{0} \text { (given) and } F\left(y_{t}\right) \leq \dot{y}_{t} \leq G\left(\bar{y}_{t}, y_{t}, x_{t}\right)\right\} .
$$

We are looking for an externality $\bar{y}$ which is a fixed-point of the correspondence 


$$
\Lambda(\bar{y})=\arg \max _{y}\left\{\int_{0}^{+\infty} u\left(x_{t}\right) e^{-r t} d t \mid(x, y) \in \Delta(\bar{y})\right\} .
$$

Indeed, in Fixed-Point Theory, the main results required the upper semicontinuity of the correspondence $\Lambda$ (here the solution exhibited by our theorem) with respect to the variable (here, the externality $\bar{y}$ ). This part is difficult to establish since there is a lack of lower semi-continuity of the correspondence $\Delta$ which prevents us to apply the Maximum Principle of Berge.

\section{Appendix}

The original Fatou's lemma has been generalized to the following proposition (See e.g. Hildenbrand and Mertens, 1971).

Proposition 1 Let $\left(h_{n}\right)$ be an integrably bounded sequence of integrable mappings from $\Omega \subset \mathbb{R}$ to $\mathbb{R}^{k}$, which converges weakly to a integrable mapping $f$ : $\Omega$ to $\mathbb{R}^{k}$. Then, we have

$$
\text { for a.e. } a \in \Omega, f(a) \in \operatorname{coLs} s_{n}\left\{f_{n}(a)\right\} \text {, }
$$

where $L s_{n}\left\{x_{n}\right\}$ denotes the set of limit points of converging subsequences of $\left(x_{n}\right)$, and $\operatorname{co}(Z)$ the convex hull of the set $Z$.

\section{References}

[1] Askenazy, Ph. and C. Le Van (1999), "A model of optimal growth strategy," Journal of Economic Theory 85, 24-51.

[2] Brezis, H. (1987), Analyse Fonctionnelle, Masson, Paris.

[3] Cass, D. (1965), "Optimum growth in an aggregative model of capital accumulation," Review of Economic Studies, 32, 233-240.

[4] Chichilnisky, G. (1981), "Existence and characterization of optimal growth paths including models with non-convexities in utilities and technologies," Review of Economic Studies, 48, 51-61.

[5] Dunford, N. and J. T. Schwartz (1967), Linear Operators, Interscience Publishers, New York. 
[6] Hildenbrand, W. and J.-F. Mertens, (1971) "On Fatou's lemma in several dimensions," Wahrsch. verw. Gebiete, 17, 151-155.

[7] Koopmans, T. C. (1965) "On the concept of optimal economic growth," The Econometric Approach to Development Planning, North Holland, Amsterdam.

[8] Lucas, R. E. (1988), "On the mechanics of economic development," Journal of Monetary Economics, 22, 3-42.

[9] Magill, M. J. P. (1981), "Infinite horizon programs," Econometrica, 49, 679-711.

[10] Rockafellar, R. T. (1970) Convex Analysis, Princeton University Press, Princeton.

[11] Romer, P. M. (1986a) "Cake eating, chattering and jumps: existence results for variational problems," Econometrica, 54, 897-908.

[12] Romer, P. M. (1986b) "Increasing returns and long-run growth," Journal of Political Economy, 94, 1002-1037.

[13] Rudin, W. (1987), Real and Complex Analysis, Mac Graw-Hill, New York. 